\title{
EXAMPLES OF NONSOLVABLE PARTIAL DIFFERENTIAL EQUATIONS
}

\author{
BY
}

\section{ROBERT RUBINSTEIN}

\begin{abstract}
Two examples of nonsolvable partial differential operators with multiple characteristics are presented. They illustrate the possibility that certain terms in the principal part may play no role in determining the solvability properties of the operator. This situation cannot occur for simple characteristics, where solvability is determined by the principal part.
\end{abstract}

1. In this note, we present nonsolvability results for the partial differential operators

$$
L u=u_{t t}+t^{n} u_{x x}+\left(i-t^{m}\right) u_{x}, \quad m \text { odd, }
$$

and

$$
L u=u_{t}-t^{n} u_{x x}+i t^{m} u_{x}, \quad n \text { even. }
$$

We will prove that (1) is not locally solvable at the origin if $n>4 m+2$, and that (2) is nonsolvable if $n>2 m+1$. Neither operator has simple characteristics, therefore the results of Nirenberg and Treves [5] do not apply.

It is important to know whether these conditions are also necessary for nonsolvability. The fundamental solution of (2) can be constructed by Fourier transforms if $n<2 m+1$; thus, this condition characterizes the local solvability of (2). Equation (1) is somewhat more complicated. However, we note that it is solvable regardless of $m$ when $n=1$. In this case, the fundamental solution of $u_{t t}+$ $t u_{x x}$, which can be constructed in terms of Airy functions, is a parametrix for any operator of the form $u_{t t}+t u_{x x}+f(t) u_{x}$.

For operators with simple characteristics, local solvability is determined by the principal part: the necessary and sufficient condition of [5] does not involve any lower order terms. This situation extends to pseudo-differential operators of order $m$, as long as no lower order term has order greater than $m-1$. Equation (1) illustrates the failure of this principle for multiple characteristics. The principal part $u_{t t}+t^{n} u_{x x}$ is easily shown to be solvable, whereas the combination $u_{t t}+$ $\left(i-t^{m}\right) u_{x}$ is nonsolvable; however, if $n$ is large enough relative to $m$, the term 
$t^{n} u_{x x}$ plays no role in determining the solvability properties of (1). A similar phenomenon occurs in (2) when $m$ is odd. In that case $u_{t}+i t^{m} u_{x}$ is nonsolvable and the addition of a term $t^{n} u_{x x}$ does not change this fact if $n$ is much larger than $m$.

Examples of nonsolvable operators with multiple characteristics have been given in Grushin [3] and Gilioli and Treves [2]. Equations (1) and (2) appear to differ from them somewhat. In particular, we do not think that Grushin's eigenvalue condition is relevant here. However, we are not prepared to suggest a condition which does include all of these cases.

The remaining sections are organized as follows: $\$ 2$ is largely heuristic, and is devoted to motivating the nonsolvability conditions. The nonsolvability proofs are given in $\S \S 3$ and 4 .

2. In this section, we will motivate the nonsolvability condition for equation (2). We will show that when $n>2 m+1$, (2) can be formally transformed into a nonsolvable pseudo-differential operator.

The Fourier transform of (2) with respect to $x$ is the ordinary differential operator

$$
\frac{d U}{d t}+\xi^{2} t^{n} U-\xi t^{m} U
$$

First consider the case when $m$ is odd. Suppose $\xi<0$, and introduce the change of variable $s=|\dot{\xi}|^{\alpha} t$ into (3). The result is

$$
|\xi|^{\alpha}\left[\frac{d U}{d s}+|\xi|^{2-(n+1) \alpha} s^{n} U+|\xi|^{1-(m+1) \alpha} s^{m} U\right] .
$$

Modifying the usual terminology in an obvious way, we will consider an expression such as (4) as the symbol of a pseudo-differential operator. If we choose $\alpha=2 /(n+1)$, the term in brackets in (4) becomes

$$
\frac{d U}{d s}+s^{n} U+|\xi|^{[n-(2 m+1)] /(n+1)} s^{m} U .
$$

If $n>2 m+1$, we may replace the covariable $\xi$ by an $\eta$ such that $|\eta|=$ $|\xi|^{[n-(2 m+1)] /(n+1)}$. (5) then becomes

$$
\frac{d U}{d s}+|\eta| s^{m} U+s^{n} U
$$

which is the symbol of the nonsolvable principal type operator

$$
u_{s}+s^{m}\left|\frac{\partial}{\partial y}\right| u+s^{n} u \text {. }
$$

When $m$ is even, this discussion is not useful because (6) is then solvable. However, suppose we first set $r=t-\xi^{-1 /(n-m)}$ with $\xi>0$. Then (3) becomes 


$$
\frac{d U}{d r}+\left[r+\xi^{-1 /(n-m)}\right]^{n} \xi^{2} U-\left[r+\xi^{-1 /(n-m)}\right]^{m} \xi U
$$

which may be expanded as

$$
\begin{aligned}
& \frac{d U}{d r}+\left[(n-m) \xi^{(n-2 m+1) /(n-m)} r\right. \\
& \left.+[1 / 2 n(n-1)-1 / 2 m(m-1)] \xi^{(n-2 m+2) /(n-m)} r^{2}+\cdots\right] U .
\end{aligned}
$$

Now let $s=\xi^{(n-2 m+2) / 3(n-m)} r$. (7) becomes

$$
\xi^{(n-2 m+2) / 3(n-m)}\left[\frac{d U}{d s}+(n-m) \xi^{(n-2 m-1) / 3(n-m)} s U+O(1) U\right]
$$

where $O(1)$ refers to terms which are bounded in $\xi$ as $\xi \rightarrow \infty$. As before, the term in brackets in (8) is the symbol of a nonsolvable operator with principal part $u_{s}+(n-m) s|\partial / \partial y| u$.

Although this discussion is only formal, these transformations are essential in the nonsolvability proofs. Thus, they have some more fundamental significance. In the first case, $m$ odd, the effect of the steps leading to (5) was to reduce the order of the original $t^{n} u_{x x}$ term to zero. This fact corresponds to the observation in $\S 1$ that this term plays no role in determining local solvability. The rescaling applied to equation (1) in $\S 3$ has the same effect. When $m$ is even and the nonsolvability condition holds, (8) shows that in a suitable region in the $t-\xi$ plane, the combination $t^{n} u_{x x}+i t^{m} u_{x}$ behaves like $i t u_{x}$ and thus causes nonsolvability. Neither situation is evident from the original form of the equation.

3. Under the assumption that $n>4 m+2$ and that $m$ is odd, we will prove that (1) is not locally solvable at the origin. Starting with the equation

$$
{ }^{t} L u=u_{t t}+t^{n} u_{x x}+\left(i+t^{m}\right) u_{x}=0
$$

we make the change of variable $s=\xi^{2 /(n+2)} t$, obtaining

$$
u_{s s}+\xi^{-2} s^{n} u_{x x}+\left(i \xi^{-4 /(n+2)}+\xi^{-2(m+2) /(n+2)} s^{m}\right) u_{x}=0 .
$$

For clarity of notation, we set $\xi^{1 /(n+2)}=\lambda$, and then look for a solution of (10) of the form $u=e^{\Phi(x, t, \lambda)}$. This substitution results in the Riccati-type equation for $\Phi$

$$
\begin{aligned}
A(\Phi)= & \left(\Phi_{s}\right)^{2}+\Phi_{s s}+\lambda^{-2(n+2)} s^{n}\left[\left(\Phi_{x}\right)^{2}+\Phi_{x x}\right] \\
& +\left(i \lambda^{-4}+\lambda^{-2(m+2)} s^{m}\right) \Phi_{x}=0 .
\end{aligned}
$$

To solve (11), we will use the following perturbation scheme: we make an initial guess $\Phi^{0}=\lambda^{\alpha} 0 \phi^{0}(s, x)$ and write 


$$
A\left(\Phi^{0}\right)=\lambda^{\beta} \psi^{0}(s, x)+O\left(\lambda^{\beta}{ }^{1}\right)
$$

where $\beta_{1}<\beta_{0}$. We then try to choose a correction to $\Phi^{0}, \Phi^{1}=\Phi^{0}+$ $\lambda^{\alpha}{ }^{1} \phi^{1}(s, x)$ where $\alpha_{1}<\alpha_{0}$, so that

$$
A\left(\Phi^{1}\right)=O\left(\lambda^{\beta_{1}}\right) .
$$

Comparing (12) and (13), we see that by adding a correction term of order less than $\alpha_{0}$ to $\Phi^{0}$, we have reduced the order of $A(\Phi)$ from $\beta_{0}$ to $\beta_{1}$. We remark that $\alpha_{0}$ is determined by the requirement that this first step be possible. If we made the wrong choice for $\alpha_{0}, A\left(\Phi^{1}\right)$ would always have the same order as $A\left(\Phi^{0}\right)$ whatever lower order correction term we added to $\Phi^{0}$.

Proceeding inductively, we attempt to construct a formal infinite sum $\Phi=$ $\Sigma_{n \geqslant 0} \lambda^{\alpha_{n}} \phi^{n}(s, x)$, with the $\alpha_{n}$ decreasing to $-\infty$, so that the coefficient of each power of $\lambda$ in $A(\Phi)$ is zero. As at the first step, $\alpha_{n}$ is determined by the requirement that $\alpha_{n+1}<\alpha_{n}$ should exist so that the order of $A\left(\Phi^{n+1}\right)$ is strictly less than the order of $A\left(\Phi^{n}\right)$.

This method is quite similar to the construction of formal exponential solutions of hyperbolic equations discussed in Flaschka and Strang [1]. The only significant difference is that the parameter $\xi$ appears in the coefficients of our equation (10). The theorem of [1] guaranteeing that the type of construction we have described is possible could no doubt be extended to this case; however, since we require $\Phi$ to have certain special properties, we will show how to compute the $\alpha_{n}$ and $\phi^{n}$ directly.

The first approximation is $\Phi^{0}=-i \lambda^{n+2} x$. Then $A\left(\Phi^{0}\right)=O\left(\lambda^{n-2}\right)$; the correction $\Phi^{1}=-i \lambda^{n+2} x+\lambda^{(n-2) / 2} \phi^{1}$ results in $A\left(\Phi^{1}\right)=\lambda^{n-2}\left(\phi_{s}^{1}\right)+$ $\lambda^{n-2}+$ lower order terms. The choice $\phi^{1}(t, x)=-i$ s thus eliminates the $O\left(\lambda^{n-2}\right)$ terms as required. Let

$$
\Phi^{2}=-i \lambda^{n+2} x-i \lambda^{(n-2) / 2} s+\lambda^{\alpha} \phi^{2} .
$$

Then

$$
\begin{aligned}
A\left(\Phi^{2}\right)= & -2 i \lambda^{n / 2-1+\alpha} \phi_{s}^{2}+\lambda^{2 \alpha}\left(\phi_{s}^{2}\right)^{2}+\lambda^{\alpha} \phi_{s s}^{2} \\
& +\lambda^{-2(n+2) s^{n}}\left[-\lambda^{2(n+2)}-2 i \lambda^{n+2+\alpha} \phi_{x}^{2}+\lambda^{2 \alpha}\left(\phi_{x}^{2}\right)^{2}+\lambda^{\alpha} \phi_{x x}^{2}\right] \\
& +i \lambda^{\alpha-4} \phi_{x}^{2}-i \lambda^{n-(2 m+2)} s^{m}+\lambda^{\alpha-2 m-4} s^{m} \phi_{x}^{2} .
\end{aligned}
$$

In $A\left(\Phi^{2}\right)$, the possible largest powers of $\lambda$ that contain $\alpha$ are then $n / 2-1+$ $\alpha$ and $2 \alpha$. Since we require $\alpha<n / 2-1$, we must take $n / 2-1+\alpha$ to be the larger power. The largest power of $\lambda$ not containing $\alpha$ is $n-(2 m+2)$; thus, to find $\alpha$ we set $n / 2-1+\alpha=n-(2 m+2)$, obtaining $\alpha=n / 2-$ $2 m-1$. The hypothesis $n>4 m+2$ insures that $\alpha>0$. The resulting equation 
for $\phi^{2}$ is $2 i \phi_{s}^{2}=-i s^{m}$; we choose

$$
\phi^{2}(s, x)=-\frac{1}{2(m+1)} s^{m+1}-1 / 2 x^{2} .
$$

Note that $m+1$ is even.

For the next approximation let $\Phi^{3}=\Phi^{2}+\lambda^{\alpha} \phi^{3}$. Then

$$
\begin{aligned}
A\left(\Phi^{3}\right)= & 1 / 4 \lambda^{n-4 m-2} s^{2 m}+\lambda^{2 \alpha}\left(\phi_{s}^{3}\right)^{2}-2 i \lambda^{n / 2-1+\alpha} \phi_{s}^{3}-\lambda^{n / 2-2 m-1+\alpha} s^{m} \phi_{s}^{3} \\
& -1 / 2 m \lambda^{n / 2-2 m-1} s^{m-1}+\lambda^{\alpha} \phi_{s s}^{3}+O(1)-i \lambda^{n / 2-2 m-5} x+i \lambda^{\alpha-4} \phi_{x}^{3} \\
& -\lambda^{n / 2-4 m-5} s^{m} x+\lambda^{\alpha-(2 m+4)} s^{m} \phi_{x}^{3} .
\end{aligned}
$$

We again find that in $A\left(\Phi^{3}\right)$ the largest exponent of $\lambda$ that contains $\alpha$ is $n / 2-1+\alpha$. Since $n>4 m+2$, the largest exponent not containing $\alpha$ is $n-4 m-2$. Setting $n / 2-1+\alpha=n-4 m-2$, we get $\alpha=n / 2-4 m-1$. $\alpha$ may be positive or negative; in any case, the equation that results for $\phi^{3}$ is $2 i \phi_{s}^{3}=1 / 4 s^{2 m}$. Thus, $\phi^{3}$ vanishes at the origin to a higher order than $\phi^{2}$, so that

$$
\operatorname{Re} \phi^{3}(s, x) \leqslant \phi^{2} .
$$

The remaining $\alpha_{n}$ cannot be determined a priori since they depend on complicated relationships betwen $n$ and $m$; therefore, we will only show how to find them inductively. Suppose we have found $\Phi^{j-1}$; setting $\Phi^{j}=\Phi^{j-1}+$ $\lambda^{\alpha} j_{\phi^{j}}$, we find that in $A\left(\Phi^{j}\right)$, the largest power of $\lambda$ containing $\alpha_{j}$ will be $n / 2-1+\alpha_{j} . \alpha_{j}$ will be determined by equating this quantity to the largest exponent of $\lambda$ not involving $\alpha_{j}$. We will denote this exponent by $M$. Note that the equation for $\phi^{j}$ will always be of the form $-i \phi_{s}^{j}=$ known quantity, so that a smooth solution for $\phi^{j}$ will always exist.

Let us investigate $A(\Phi)$ to determine the possible values of $M$. First, $M$ may occur in $\left(\Phi_{s}\right)^{2} . \phi^{j}$ would then vanish at the origin to an order greater than $\phi^{2}$, and (14) holds with $j$ replacing 3 . The largest power that ever appears in $\Phi_{s s}$ is less than $n / 2-1$; if this turns out at any step to be $M$, then $\alpha_{j} \leqslant 0$. Similarly, the $\left[\left(\Phi_{x}\right)^{2}+\Phi_{x x}\right]$ term does not contain any exponent greater than zero; when one of these exponents is $M$, we have $\alpha_{j} \leqslant 1-$ $n / 2<0$. Finally, in $\Phi_{x}$ the terms with the exponents $n-2$ and $n-$ $(2 m+2)$ have already been cancelled; the remaining possibilities for $M$ are less than $n / 2-2 m-3$. For these possibilities, $\alpha_{j}$ would again be negative.

Thus, we have found that the construction of $\Phi$ is indeed possible. Furthermore, we have found the following property of $\Phi$ : when $\alpha_{j}>0$, $\operatorname{Re} \phi^{j}$ vanishes at the origin to a higher order than $\phi^{2}$. Therefore, since $\alpha_{j}$ 
will only be positive a finite number of times, for any $N$

$$
\operatorname{Re} \Phi^{N}(x, s, \lambda) \leqslant-\lambda^{n / 2-2 m-1}\left[\frac{1}{2(m+1)} t^{m+1}+1 / 2 x^{2}\right]
$$

holds in some neighborhood of the origin. Next, note that the construction of $\Phi$ implies that if any integer $K$ is given, an $L$ exists so that

$$
{ }^{t} L \exp \Phi_{N}(x, s, \lambda)=O\left(\lambda^{-K}\right) .
$$

(15) and (16) imply that $L$ is nonsolvable. The proof follows Hörmander's argument in [4]. If $L$ is locally solvable at the origin, the inequality

$$
\begin{aligned}
& \left|\iint_{\Omega} u(x, t) f(x, t) d x d t\right| \\
& \quad \leqslant C \sup \sum_{|\alpha|<N}\left|D^{\alpha} f(x, t)\right| \cdot \sup \sum_{|\alpha| \leqslant N}\left|D^{\alpha}{ }^{t} L u(x, t)\right|
\end{aligned}
$$

must hold. In (17), $\Omega$ is some neighborhood of the origin, and $u, f \in C_{0}^{\infty}(\Omega)$. Let $\phi(s, x)$ be a cutoff function which is identically one near the origin and is supported in the open set where (15) holds. Since $s=\xi^{2 /(n+2)} t$, the support of $\phi(s, x)$ is contained in $\Omega$ for all large $\xi$. Now define $u(t, x)=$ $\phi(s, x) \exp \Phi_{N}(s, x, \lambda)$. Following Hörmander, we conclude from (15) and (16) that (17) cannot hold. Thus, $L$ is nonsolvable. We refer to [4] for the details of the argument.

4. In this section, we consider equation (2). First, we prove nonsolvability when $m$ is odd and $n>2 m+1$. The argument is similar to that of $\S 3$, so we will only discuss the main points in the proof.

Beginning with the equation

$$
-{ }^{t} L u=u_{t}+t^{n} u_{x x}-i t^{m} u_{x}=0
$$

we make a rescaling transformation $s=\xi^{2 /(n+1)} t$, obtaining

$$
u_{s}+s^{n} \xi^{-2} u_{x x}-i s^{m} \xi^{-2(m+1) /(n+1)} u_{x}=0 .
$$

Setting $u=e^{\Phi(x, t, \lambda)}$ with $\lambda^{n+1}=\xi$, (19) becomes

$$
\Phi_{s}+s^{n} \lambda^{-2(n+1)}\left[\left(\Phi_{x}\right)^{2}+\Phi_{x x}\right]-i s^{m} \lambda^{-2(m+1)} \Phi_{x}=0 .
$$

(20) is solved exactly as (11). For $\Phi^{1}$, we may choose

$$
i \lambda^{n+1} x-\lambda^{n-(2 m+1)}\left[\frac{1}{m+1} s^{m+1}+1 / 2 x^{2}\right] \text {. }
$$

As in $\S 3$, we cannot determine the further powers of $\lambda$ a priori; but, we can conclude that 


$$
\operatorname{Re} \Phi^{N}(x, t, \lambda) \leqslant-\lambda^{n-(2 m+1)}\left[\frac{1}{m+1} t^{m+1}+1 / 2 x^{2}\right] .
$$

Since $n>2 m+1$ and $m$ is odd, (21) contradicts (17). We conclude that $L$ is nonsolvable.

A different proof is needed for nonsolvability when $m$ is even. We make the change of variable $t=\lambda^{-(n-2 m+2) / 3} s-1 / \lambda$. After setting $u=e^{\Phi(x, t, \lambda)}$, (18) becomes

$$
\begin{aligned}
A(\Phi)= & \Phi_{s}+\lambda^{-(n-2 m+2) / 3}\left[\lambda^{-(n-2 m+2) / 3} s-1 / \lambda\right]^{n}\left\{\left(\Phi_{x}\right)^{2}+\Phi_{x x}\right\} \\
& -i \lambda^{-(n-2 m+2) / 3}\left[\lambda^{-(n-2 m+2) / 3} s-1 / \lambda\right]^{m} \Phi_{x}=0 .
\end{aligned}
$$

We begin with the first approximation $\Phi^{0}=i \lambda^{n-m} x$. Then

$$
\begin{aligned}
A\left(\Phi^{0}\right) & =(n-m) \lambda^{(n-2 m-1) / 3} s-\left[\frac{n(n-1)}{2}-\frac{m(m-1)}{2}\right] s^{2} \\
& +O\left(\lambda^{-(n-2 m-1) / 3}\right) .
\end{aligned}
$$

Now let $\Phi^{1}=i \lambda^{n-m} x+\lambda^{(n-2 m-1) / 3} \phi^{1}$. The resulting equation for $\phi^{1}$ is $\phi_{s}^{1}=-(n-m) s$, so we may take $\phi^{1}(x, s)=-1 / 2(n-m) s^{2}-1 / 2 x^{2}$. For the next approximation $\Phi^{2}=\Phi^{1}+\lambda^{\alpha} \phi^{2}$, we find that $\alpha \leqslant 0$. Therefore

$$
\operatorname{Re} \Phi^{N}(x, s, \lambda) \leqslant-\lambda^{(n-2 m-1) / 3}\left[\frac{1}{2}(n-m) s^{2}+1 / 2 x^{2}\right] .
$$

Since $n>2 m+1,(22)$ implies that $L$ is nonsolvable.

\section{REFERENCES}

1. H. Flaschka and G. Strang, The correctness of the Cauchy problem, Advances in Math. 6 (1971), 347-379. MR 43 \#5147.

2. A. Gilioli and F. Trèves, An example in the solvability theory of linear partial differential equations (to appear).

3. V. V. Grušin, On a class of elliptic pseudodifferential operators degenerate on a submanifold, Mat. Sb. 84 (126) (1971), 163-195 = Math. USSR Sb. 13 (1971), 155-1 86.

MR 44 \#860.

4. L. Hörmander, Linear partial differential operators, Die Grundlehren der math. Wissenschaften, Band 116, Academic Press, New York; Springer-Verlag, Berlin, 1963, Chap. 6 MR 28 \#4221.

5. L. Nirenberg and F. Trèves, On local solvability of linear partial differential equations, Comm. Pure Appl. Math. 23 (1970), 1-38, 459-509. MR 41 \#9064a, b.

DEPARTMENT OF MATHEMATICS, UNIVERSITY OF UTAH, SALT LAKE CITY, UTAH 84112 